\title{
Catheter Site
}

National Cancer Institute

\section{Source}

National Cancer Institute. Catheter Site. NCI Thesaurus. Code C92596.

The anatomic site through which fluid is transferred into or out of the body using a catheter. 